
rural

\title{
Doit-on et peut-on éviter la grande évacuation des campagnes africaines et asiatiques?
}

Should we and can we avoid a major migration from African and Asian rural areas?

\section{Henri Rouillé d'Orfeuil}

\section{(2) OpenEdition} Journals

\section{Édition électronique}

URL : http://journals.openedition.org/economierurale/2254

DOI : $10.4000 /$ economierurale.2254

ISSN : 2105-2581

Éditeur

Société Française d'Économie Rurale (SFER)

Édition imprimée

Date de publication : 10 septembre 2007

Pagination : 124-128

ISSN : 0013-0559

Référence électronique

Henri Rouillé d'Orfeuil, « Doit-on et peut-on éviter la grande évacuation des campagnes africaines et asiatiques? », Économie rurale [En ligne], 300 | Juillet-août 2007, mis en ligne le 12 novembre 2009, consulté le 01 mai 2019. URL : http://journals.openedition.org/economierurale/2254 ; DOI : 10.4000/ economierurale.2254 


\title{
Doit-on et peut-on éviter la grande évacuation des campagnes africaines et asiatiques ?
}

\author{
Henri ROUILLÉ d'ORFEUIL \\ Président de Coordination SUD
}

\begin{abstract}
L'agriculture est multifonctionnelle. Il appartient donc aux responsables politiques de fixer les priorités et les synergies entre les diverses fonctions de l'agriculture pour définir une politique compatible avec les attentes des électeurs-citoyens. Dans ce texte, il ne s'agit pas de développer un raisonnement sur l'économie politique agricole. L'objectif d'Henri Rouillé d'Orfeuil est d'alerter les économistes agricoles et ruraux sur l'un des plus grands défis qu'il faudra prendre en compte dans un proche avenir : celui du travail paysan. À partir de données précises, l'inégale répartition des paysans entre les régions du monde est d'abord exposée. Elle permet de comprendre ensuite les hémorragies paysannes et l'exclusion du monde rural auxquelles l'humanité n'échappera pas.
\end{abstract}

$\mathbf{L}^{\mathrm{s}}$ es agricultures remplissent plusieurs fonctions. Elles sont par nature multifonctionnelles, même si parfois elles oublient de s'intéresser à certaines de leurs fonctions. Elles doivent produire des aliments en volume suffisant, fournir aux consommateurs des produits de qualité, contribuer à la bonne gestion des ressources naturelles et à la conservation ou à la création d'un environnement de qualité, participer à l'aménagement du territoire, produire de l'énergie, qu'il s'agisse traditionnellement de bois de feu ou, plus récemment, d'agrocarburants. Enfin, elles représentent toujours une petite moitié du travail humain mondial et constituent l'activité économique de loin la plus employeuse. Elles permettent à cette moitié d'humanité de survivre, parfois de vivre.

Cette multifonctionnalité nécessite que les multiples objectifs et logiques de chacune de ces fonctions soient rendues compatibles. Cette compatibilité n'est pas naturelle. Il n'y a pas nécessairement win-win, selon l'expression de nos partenaires anglo- saxons. On observe même que certains types d'agricultures ignorent sciemment certaines fonctions, voire ne se préoccupent que d'une seule d'entre elles, celle par exemple de produire en quantité des produits agricoles, que ceux-ci soient alimentaires, industriels ou énergétiques. Le marché, loin de favoriser une compatibilité entre les différentes fonctions, a tendance à spécialiser les exploitations agricoles, voire les régions ou les pays. Cette absence d'autorégulation rendent indispensable les politiques agricoles qui, pour l'essentiel, tout en jouant avec les marchés, doivent s'efforcer d'articuler des incitations et des désincitations, voire des interdits pour amener les agriculteurs à avoir des comportements producteurs d'intérêt général. Dans un contexte donné et dans un débat avec les parties prenantes organisées et, plus largement, avec l'ensemble des citoyens-électeurs, il appartient aux responsables politiques de fixer les priorités et les synergies entre les fonctions et de définir une politique agricole. Évidemment, ce serait plus facile s'il y avait win-win ou 
si on trouvait la solution miracle permettant à toutes les fonctions d'être optimisées par les mêmes mesures de politique agricole. Ce n'est pas le cas, il y a des contradictions qui nécessitent que des choix soient faits.

\section{La disparition du travail paysan L'avenir incertain des producteurs agricoles}

Mon propos, dans ce texte personnel, n'est pas de développer ici un raisonnement sur la recherche de cet optimum général, ce sera sans doute fait par ailleurs tant cette question est au cour de l'économie politique agricole, mais d'attirer l'attention sur l'une de ces fonctions, celle qui intéresse le moins les responsables : la prise en compte du travail humain.

Les paysans représentent toujours environ $43 \%$ du travail humain. Ce travail diffère considérablement d'une région et d'une agriculture à une autre. Dans les pays développés, les agriculteurs représentent en général moins de $5 \%$, le plus souvent entre 2 et $3 \%$ des actifs. À l'autre extrême, dans la plupart des pays d'Asie et d'Afrique, les paysans sont encore $60 \%$ à $70 \%$ de la population. Pour comprendre l'inégale répartition des paysans entre les régions du monde, il faut lire 1'histoire, celle de la colonisation, celle de la collectivisation, celle de la modernisation et celle de la libéralisation.

S'agissant de la colonisation, il convient de distinguer les colonisations entre celles qui ont confisqué et concentré la terre (Amérique du Nord et du Sud, Afrique australe, Océanie et quelques pays d'Asie où l'on retrouve des agricultures latifondiaires, Europe du Sud et de l'Est) et celle qui n'ont pas perturbé le foncier (Afrique subsaharienne et Asie).

Des processus de collectivisation, autre rupture brutale avec les histoires agraires, se sont imposés en Europe de l'Est et dans les pays communistes du tiers-monde.
La modernisation de l'économie et, en conséquence de l'agriculture, s'est manifestée partout mais plus ou moins précocement.

\section{Résultats : en Amérique, en Afrique australe et en Océanie...}

Les agricultures des régions à colonisation foncière ont depuis leurs origines des structures de production, des relations capital/ travail et des orientations agro-exportatrices que n'ont pas transformées les indépendances. Les terres n'ont pas été rendues aux peuples qui les possédaient ou qui en jouissaient avant la colonisation ni même été redistribuées aux citoyens de ces nouveaux pays. Les colons, en grande partie propriétaires fonciers, se sont emparés du pouvoir et ont imposé un nouveau cadre politique. Certes, le développement des économies nationales a interféré différemment avec ce schéma aux États-Unis, au Brésil, en Afrique du Sud ou en Australie, le rapport capital/travail a évolué, notamment avec la fin légale de l'esclavage, de la ségrégation ou de l'apartheid, mais nous sommes toujours dans un même schéma et les agricultures de ces pays se retrouvent dans le groupe de Cairns autour de positions et de revendications communes lors des rounds de la négociation commerciale. Ces pays ont nourri leurs métropoles, ils se proposent de nourrir le monde.

\section{Résultats : en Afrique et en Asie...}

Il en va différemment en Afrique et en Asie, continents où les puissances coloniales ont peu touché au foncier, même si partout on retrouve des agricultures de plantation. Les paysanneries ont traversé les siècles, vivant souvent en marge des processus de modernisation, même si elles ont largement contribué aux courants d'échanges de produits agricoles, aux recettes fiscales et à l'investissement humain dans des travaux d'infrastructure. Cette petite vie économique a permis à ces paysanneries, accrochées à leurs terres, de subsister et de rester aujourd'hui encore largement majoritaires. Les 
exploitations familiales constituent en effet des unités qui sont à la fois des unités de production, de consommation, de gestion des ressources, d'emploi, de solidarité. Mais, cette petite vie est aujourd'hui violemment agressée par un processus de libéralisation et par une forme de modernisation imaginée pour d'autres agricultures. Ces deux processus organisent une compétition commerciale mondiale, si ce n'est une guerre, entre les différentes agricultures du monde, une guerre entre combattants dont les armes sont tout à fait inégales. Il s'agit d'une compétition pour les marchés, y compris domestiques, mais aussi pour les ressources nécessaires à la production agricole, à commencer par la terre. Les paysanneries sont mises à mal, même si la montée des prix agricoles ces derniers mois apporte à certains un peu d'oxygène. Le salut par la libéralisation et par une forme de modernisation conçue pour les agricultures du nord du monde, prônées par Centres internationaux de recherche et par les tuteurs politicoéconomiques des pays concernés, principalement par les institutions financières internationales, mais aussi par les puissances économiques, qui se nourrissent de cette modernisation à base de produits chimiques, mécaniques et biologiques, renforce la fracture qui existe au sein des mondes agricoles. Même si une proportion des exploitations agricoles, très minoritaire à l'échelle mondiale, peut s'inscrire dans cette compétition, la très grande majorité des paysans n'y résiste pas. Les paysanneries sont alors entraînées dans des processus de paupérisation, souvent d'endettement et d'hypothèque, lorsqu'il y a petite propriété des terres et, à terme, d'exclusion. Et si les mêmes facteurs entraînent les mêmes résultats, il y a tout lieu de penser que le processus de paupérisation/exclusion ne s'arrêtera que lorsqu'il n'y aura plus que quelques pourcents d'agriculteurs.

Passer de 43 à $3 \%$ de paysans signifie que seulement 0,2 milliard de paysans réussiront à rester dans la production agricole contre aujourd'hui encore 2,9 milliards de paysans. Ce sont donc 2,7 milliards de femmes, d'hommes et d'enfants qui seront amenés à quitter l'agriculture par la petite porte, celle de la pauvreté et de l'endettement. Ce chiffre, établi à démographie constante ce qui est très optimiste - pourrait être atteint en une ou deux décennies si la libéralisation venait à être menée tambour battant comme souhaitent l'imposer les puissances économiques. Il est insoutenable pour une économie mondiale qui doit déjà réinclure des centaines de millions, voire un ou deux milliards de personnes qui vivent déjà en situation d'exclusion. Nous reviendrons sur ce qui est sans doute l'une des variables les plus essentielles du tableau de bord de l'histoire contemporaine : le ratio entre la vitesse d'exclusion agricole et la vitesse, ou les capacités, d'inclusion dans d'autres secteurs de l'économie.

\section{Résultats :...dans les pays du Nord}

La modernisation des agricultures associée à la capacité d'inclusion de travail hors agriculture a permis d'aller vers ce pourcentage de 2 ou $3 \%$ de producteurs agricoles. Le cheminement a certes été différent selon qu'il partait d'agricultures coloniales non décolonisées ou d'agricultures paysannes et/ou familiales. Les structures de production restent différentes mais, dans tous les cas, la production agricole n'est plus employeuse majoritaire. S'agissant de nos agricultures familiales d'Europe de l'Ouest, ce passage de $60 \%$ à $3 \%$ s'est fait sur un bon siècle et dans un contexte tout à fait différent de celui dans lequel une telle évolution du travail agricole aurait à se produire en Asie et en Afrique. Cette régression du travail agricole, qui s'est manifestée concrètement par des départs vécus souvent douloureusement, a été rendue possible par une série de facteurs qui n'existent plus aujourd'hui : l'émigration de près de soixante millions d'Européens, dont beaucoup de paysans, vers les pays dits du nouveau monde à la jointure des $\mathrm{XIX}^{\mathrm{e}}$ et $\mathrm{XX}^{\mathrm{e}}$ siècles, deuX guerres 
mondiales particulièrement meurtrières pour les ruraux, des périodes de croissance fondée sur le développement d'industries fortement créatrices d'emplois, une école de la République permettant à des générations de jeunes paysans de quitter la terre et d'aller vers des emplois non agricoles, le développement d'activités para-agricoles et d'industries agro-alimentaires réparties sur tout le territoire, enfin l'existence d'États dotés de politiques sociales permettant de gérer la transition et faciliter, sinon de prendre en charge, les parcours individuels de migration économique. Les transferts d'emplois de plus de la moitié de la population française se sont réalisés sans que ne se crée une population durablement exclue. Ce n'est que dans les années 1970 qu'un chômage structurel a fait son apparition et a atteint dans notre pays $10 \%$ des actifs. Peu de ces conditions se rencontrent aujourd'hui dans les continents où des hémorragies humaines sont sur le point de se produire.

\section{Libéralisation et hémorragie paysannes}

Si la modernisation des agricultures, sous la forme qui a été la sienne, a joué un grand rôle dans les évolutions que nous venons de décrire, la libéralisation des échanges de produits et des marchés internationaux a eu également un impact majeur ces dernières décennies. L'ampleur de ce processus d'ouverture des marchés se décide dans les négociations internationales, se détermine dans la définition des politiques macroéconomiques ou, dans les pays pauvres et endettés, s'impose dans la négociation des politiques commerciales lorsque les pays sont obligés de donner des contreparties et de satisfaire aux conditionnalités fixées par les institutions financières internationales lorsque celles-ci négocient des programmes d'aide ou des annulations de dettes. La mise en compétition d'agricultures dont les productivités et les compétitivités se situent dans des rapports de 1 à 100, voire de 1 à 1000 donne des résultats que l'on peut prévoir : la disparition des agricultures paysannes et familiales qui perdent leurs marchés et l'accès à leurs facteurs de production, dans la mesure où ceux-ci ou ceux-là ont quelque intérêt économique et commercial. Nous savons que la réduction de tous les obstacles à l'intégration des marchés locaux et nationaux de produits et de facteurs de production permet un optimum théorique indépassable. Le modèle classique prône sans le dire la création d'une immense ferme mondiale où toutes ressources humaines, naturelles, technologiques et financières pourraient se combiner sans contraintes.

Étudiants de l'Institut national agronomique $^{1}$, nous avions fait ce petit calcul pour un village du Cambrésis : le modèle proposait, bien sûr, la fusion des 27 exploitations agricoles en une seule unité ! Heureusement, bien d'autres facteurs, oubliés par le modèle, interviennent. Mais quand même l'ouverture des marchés sans précaution dans un contexte de guerre commerciale, notamment si l'agriculture redevient une activité rentable, condamne des exploitations agricoles dont les armes économiques sont dérisoires.

$\mathrm{Si}$, par malheur, les centaines de millions d'exploitations agricoles -on entend en général le chiffre de 1,3 milliard- étaient exposées sans protection aucune aux quelques dizaines de millions d'exploitations armées pour la guerre commerciale, un processus d'exclusion, aujourd'hui encore timide par rapport à ce qu'il pourrait devenir, s'accélérerait.

Que faire contre des compétiteurs bénéficiant d'un environnement et d'infrastructures construites pour cette bataille, disposant d'accès à des informations, à des services de recherche, à des services financiers, soutenus par des services publics de qualité et appuyés par des politiques agricoles dédiées aux seuls agriculteurs

1. AgroParisTech, aujourd'hui. 
nationaux, sans parler des structures même de ces exploitations qui permet à ces exploitants de résister aux chocs ? Les hémorragies pourraient alors concerner quelques 800 millions de paysans chinois, 700 millions d'Indiens... et au total quelques 2,7 milliards de paysans hommes, femmes et enfants. Face à de telles hémorragies, qu'elle a fini par reconnaître après avoir fait l'apologie d'agricultures de niches au premier rang desquels se trouvent toujours les fleurs coupées du Kenya ou de Colombie, la Banque mondiale demande le développement de grands programmes de formation professionnelle pour atteindre les emplois de la nouvelle croissance chinoise ou indienne, et de citer les call centers ou la silicon valley de Bangalore ou de célébrer le microcrédit et la création de micro-activités économiques. Certes, mais de là à imaginer la ré-inclusion de 2,7 milliards de personnes, s'ajoutant aux quelques 1,5 milliard d'exclus déjà en attente de réinsertion, le tout sans prendre en compte la croissance démographique, c'est parfaitement irréaliste.

Nous savons qu'il y a un mouvement historique de transfert de l'agriculture vers les autres secteurs, de la campagne vers la ville. Nous savons qu'il est irréaliste de chercher à figer les hommes dans leurs secteurs d'activités. Mais de là à ignorer la question de la réinsertion des exclus est parfaitement irresponsable. C'est pourtant ce qui se passe. Bien peu de monde s'inquiète de ce rapport totalement déséquilibré entre les vitesses d'exclusion et d'inclusion. Quels responsables politiques évoquent cette terrible question dans les négociations internationales? Comment est-elle prise en compte à l'OMC ? Qu'en dit la Banque mondiale? Quels syndicats s'occupent sérieusement de ce grand écrémage ? Quels experts introduisent dans leurs modèles ce qui pourrait apparaître comme une contrainte? L'introduction dans le modèle macroéconomique d'équilibre général de la Banque mondiale de quelques éléments sur le marché du travail, comme l'a fait le Carnegie Endowment, l'un des plus fameux think tank américains, a fait fondre les avantages tirés par les différents pays de la libéralisation. Enfin, l'hypothèse, qui est celle de ce modèle de référence, d'un plein-emploi généralisé a pu être questionnée et l'exclusion paysanne a pu cesser d'être considérée comme une bénédiction économique.

$$
\text { * }
$$

Je me souviens d'une mission dans la plaine indo-gangétique réalisée en 1998 avec un expert de l'un des centres internationaux de recherche agricole. Son diagnostic fut rapide, clair et net : «Rien n'est possible si les exploitations agricoles n'atteignent pas 15 hectares ».

Question :

«Très bien, mais les exploitations familiales font moins d'un hectare, alors que vont faire les 14 familles qui vont devoir quitter?

Conclusion tout aussi claire :

Ce n'est pas mon problème. »

De fait, ce n'est le problème de personne : grande irresponsabilité des experts et des autorités politiques face à cette question de l'exclusion agricole, que l'on cherche souvent à confondre avec un processus d'urbanisation. C'est pourtant l'une des grandes questions de notre histoire contemporaine qui pourrait provoquer des drames incommensurables et couper définitivement l'humanité en deux, entre le monde des inclus et celui des exclus, et transformer la planète en poudrière.

Messieurs les économistes, même si votre champ principal d'investigation est agricole et rural, prenez, s'il vous plaît, cette question de l'exclusion paysanne très au sérieux. 